\title{
Correction to: Resonance in Physiologically Structured Population Models
}

\author{
Kevin Gross ${ }^{1}$ (D) André M. de Roos ${ }^{2,3}$ (ID \\ Published online: 30 August 2021 \\ (C) Society for Mathematical Biology 2021
}

\section{Correction to: Bulletin of Mathematical Biology (2021) 83:86 https://doi.org/10.1007/s11538-021-00915-2}

"The original version of this article unfortunately contained mistakes in supplementary materials that were introduced during production. This erratum corrects those mistakes by providing appropriate file names for the R Supplementary files and including three missing Supplementary plain text files."

The correct versions of ESMs are updated in this correction.

The original article has been corrected.

Supplementary Information The online version contains supplementary material available at https://doi. org/10.1007/s11538-021-00931-2.

Publisher's Note Springer Nature remains neutral with regard to jurisdictional claims in published maps and institutional affiliations.

The original articles can be found online at https://doi.org/10.1007/s11538-021-00915-2.

Kevin Gross

krgross@ncsu.edu

1 Department of Statistics, North Carolina State University, Raleigh, NC 27695, USA

2 Institute for Biodiversity and Ecosystem Dynamics, University of Amsterdam, Amsterdam, The Netherlands

3 Santa Fe Institute, Santa Fe, NM 87501, USA 